\title{
Optimizing the Processing Conditions for the Reinforcement of Epoxy Resin by Multiwalled Carbon Nanotubes
}

\author{
S. Arun, Mrutyunjay Maharana, and S. Kanagaraj \\ Department of Mechanical Engineering, Indian Institute of Technology Guwahati, Guwahati 781039, India \\ Correspondence should be addressed to S. Kanagaraj; kanagaraj@iitg.ernet.in
}

Received 31 May 2013; Accepted 6 July 2013

Academic Editor: Guifu Zou

Copyright ( 92013 S. Arun et al. This is an open access article distributed under the Creative Commons Attribution License, which permits unrestricted use, distribution, and reproduction in any medium, provided the original work is properly cited.

\begin{abstract}
The reinforcement of epoxy by MWCNTs is done to obtain the required properties of composites. However, the homogeneous dispersion of MWCNTs in epoxy is a critical problem. Hence, an attempt is made to optimize the processing conditions for dispersing the MWCNTs in epoxy by solvent dispersion technique. The epoxy/MWCNTs mixture was prepared using three methods: (1) magnetic stirring at $55^{\circ} \mathrm{C}$, (2) hot air oven process at $55^{\circ} \mathrm{C}$, and (3) vacuum oven process at room temperature. The nanocomposites having 0.1 and $0.2 \mathrm{wt} . \%$ of MWCNTs were prepared, for each method. The mechanical properties of nanocomposites were studied as per ASTM-D695, and the thermal conductivity was measured using KD2 probe. It is observed that the compressive strength, Young's modulus, and thermal conductivity of $0.2 \mathrm{wt} . \%$ of MWCNTs prepared by vacuum oven method were found to be enhanced by $39.4,10.7$, and 59.2\%, respectively, compared to those of pure epoxy. Though the properties of nanocomposites were increased with MWCNTs' concentration irrespective of the processing techniques, the vacuum-processed sample showed the most enhanced properties compared to any other method. It is concluded that a unique method for the dispersion of MWCNTs in epoxy is the solvent dispersion technique with vacuum drying process.
\end{abstract}

\section{Introduction}

The usage of nanoparticles as the reinforcement in a polymer matrix is increased enormously to achieve the required properties of composites. Among all the fillers such as $\mathrm{TiO}_{2}, \mathrm{ZrO}_{2}$, and nanoclay, multiwalled carbon nanotubes (MWCNTs) were paid a lot of attention because of their attractive mechanical properties which were reported by Gojny et al. [1]. Good aspect ratio of MWCNTs with the specific surface area of $1300 \mathrm{~m}^{2} / \mathrm{g}$ helps in effective stress transfer from the matrix, which was studied by Yu et al. [2]. The desirable properties of MWCNTs made them a candidate for reinforcing the polymer matrix. Kim et al. [3] found that the homogenous dispersion of the MWCNTs which gives good interaction between the matrix and the reinforcement must be ensured, in order to achieve the improved properties of epoxy/MWCNTs nanocomposites. Starkova et al. [4] also evaluated the mechanical properties of the epoxy-based composites. The enhancement of Young's modulus of $0.1 \mathrm{wt} . \%$ composites was found to be $2 \%$ compared to that of pure epoxy. Mahfuz et al. [5] studied the influence of MWCNTs in epoxy, and it was found that the mechanical and thermal properties of the composites were increased. It was observed by He and Tjong [6] that the homogeneous dispersion of reinforcement was confirmed with an increase of electrical conductivity of the composites. It is observed from the previous studies that the dispersion of reinforcement is one of the most important factors in deciding the enhancement of mechanical, thermal, and electrical properties of composites.

Apart from the dispersion, the functionalization of MWCNTs is also an important factor. The functionalization of MWCNTs increased the stress transfer at the interface of the matrix and MWCNTs, and it also helped to reduce the concentration of substrate metallic particles present in the MWCNTs. The attachment of amine group, carboxyl group, and ozone-treated MWCNTs was studied in order to ensure the homogeneous dispersion of the MWCNTs in matrix by Ma et al. [7]. Buang et al. [8] observed that the usage of acid-treated MWCNTs showed homogeneous dispersion and less defects on the side walls of MWCNTs compared to other functionalization techniques. Theodore et al. [9] studied the effect of various functionalized MWCNTs in 
TABLE 1: Different methods for MWCNTs reinforcement.

\begin{tabular}{lcccc}
\hline Sample & MWCNTs wt.\% & Acetone + MWCNTs & Epoxy + acetone & Removal of acetone after mixing \\
\hline A-H 0.1 & 0.1 & Tip sonication & Bath sonication & Hot air oven at $55^{\circ} \mathrm{C}$ \\
A-H 0.2 & 0.2 & Tip sonication & Bath sonication & Hot air oven at $55^{\circ} \mathrm{C}$ \\
B-M 0.1 & 0.1 & Tip sonication & Bath sonication & Magnetic stirring at $55^{\circ} \mathrm{C}$ \\
B-M 0.2 & 0.2 & Tip sonication & Bath sonication & Magnetic stirring at $55^{\circ} \mathrm{C}$ \\
C-V 0.1 & 0.1 & Tip sonication & Bath sonication & Vacuum oven at RT \\
C-V 0.2 & 0.2 & Tip sonication & Bath sonication & Vacuum oven at RT \\
\hline
\end{tabular}

terms of thermal and mechanical properties. It was concluded that the attachment of $-\mathrm{COOH}$ group on the side walls of MWCNTs confirmed the enhancement of flexural strength and flexural modulus by 25.5 and $54.8 \%$, respectively, for the 1 wt.\% of reinforcement. Yu et al. [10] evaluated that the fracture toughness of epoxy reinforced with 1 and 3 wt.\% of MWCNTs was found to be enhanced by 1.29 and 1.62 times, respectively. Similarly, the fatigue life was found to be enhanced by 9.3 and 10.5 times for the same MWCNTs concentration. Rahman et al. [11] optimized the mechanical properties of the epoxy reinforced with E-glass and aminofunctionalized MWCNTs. The strength, Young's modulus, and strain at fracture of epoxy/E-glass/0.3 wt.\% of MWCNTs were found to be enhanced by 37,21 , and $21 \%$, respectively. It is observed from the previous studies that the functionalization of MWCNTs is very much essential in order to utilize the fullest potential of the reinforcement.

The thermal conductivity of the epoxy nanocomposites having 0.5 and 1 wt.\% of silica-coated MWCNTs was found to be enhanced by 51 and $67 \%$, respectively, by Cui et al. [12]. The interfacial bonding between epoxy and MWCNTs was also confirmed by the increase of thermal conductivity and broadening of the glass transition temperature (Tg) in the study by Pillai and Ray [13]. The MWCNTs were aligned in the epoxy resin to achieve the required properties by Park et al. [14], and the thermal conductivity of epoxy/MWCNTs nanocomposites at room temperature (RT) was observed to be $55 \mathrm{~W} / \mathrm{mK}$, and the stretched MWCNTs-epoxy sheet showed the value of $100 \mathrm{~W} / \mathrm{mK}$, whereas the same for pure epoxy was found to be $0.11 \mathrm{~W} / \mathrm{mK}$ at RT.

Though different types of research works are going on in the field of epoxy-based nanocomposites, the homogeneous dispersion of reinforcement is yet to be achieved, and the unique way for the dispersion of MWCNTs in epoxy remains unfulfilled. Hence, an attempt is made to optimize the processing parameters in order to improve the mechanical and thermal properties of nanocomposites.

\section{Materials and Methods}

2.1. Materials. The MWCNTs were purchased from $\mathrm{M} / \mathrm{s}$ Shenzhen Nanotech Port Co., Ltd., China. The specifications of as-received MWCNTs are as follows: outer diameter $<10 \mathrm{~nm}$, length $5-15 \mu \mathrm{m}$, purity $97 \%$, ash content $<3 \%$, and specific surface area -250 to $300 \mathrm{~m}^{2} / \mathrm{g}$. Epoxy resin and hardener were received from $\mathrm{M} / \mathrm{s}$ Endolite, India, Inc., having the density of 2.25 and $0.94 \mathrm{~g} / \mathrm{mL}$, respectively.
2.2. Chemical Treatment and Characterization of MWCNTs. The MWCNTs were functionalized using the acid treatment technique as suggested by Esumi et al. [15], which is briefly discussed here. The MWCNTs were dispersed in nitric and sulfuric acid mixture having the volume ratio of $1: 3$ and heated at $140^{\circ} \mathrm{C}$ in an oil bath with continuous mechanical stirring for $30 \mathrm{~min}$. After the chemical treatment, MWCNTs were washed with distilled water until the $\mathrm{pH}$ value of the supernatant reached around 7. Then, the MWCNTs were dried in a hot air oven at $100^{\circ} \mathrm{C}$. Thus, the chemically treated MWCNTs were obtained. The functional groups attached on the side walls of the MWCNTs were confirmed by the Fourier transform infrared spectroscopy (FTIR) technique. The concentration and types of functional groups present on the MWCNTs depend on the time of reflux and acid strength. Motchelaho et al. [16] reported that the peaks identified at 1360,1710 , and $3403 \mathrm{~cm}^{-1}$ were confirmed to be COO-, $\mathrm{C}=\mathrm{O}$, and $-\mathrm{OH}$ bonds, respectively, in the chemically modified MWCNTs. Peaks at 1710 and $3453 \mathrm{~cm}^{-1}$ were attributed to acidic groups like carbonyl, phenol, and lactol. Peak at $1576 \mathrm{~cm}^{-1}$ was assigned to $\mathrm{C}=\mathrm{C}$ bond in MWCNTs. The defects in the MWCNTs after the chemical treatment were studied by the laser micro-Raman with $488 \mathrm{~nm}$ blue laser, where the defects were observed to be negligible.

\subsection{Preparation of Nanocomposites. The resin and hardener} having the weight ratio of 1:0.4 were hand-mixed using a stirrer rod for $15 \mathrm{~min}$. and poured into the die having the dimension of $50 \mathrm{~mm}$ length and $9 \mathrm{~mm}$ diameter. Then, the mold was allowed to cure at $26 \pm 2^{\circ} \mathrm{C}$ for $3 \mathrm{hrs}$, and, thus, the pure epoxy specimen was obtained. The nanocomposites having 0.1 and $0.2 \mathrm{wt} . \%$ of MWCNTs were prepared by three methods, and their detailed specifications are given in Table 1.

The epoxy resin was dissolved in acetone using bath sonication for $30 \mathrm{~min}$., and the MWCNTs were dispersed in acetone using tip sonication for $30 \mathrm{~min}$. Both were mixed together and bath-sonicated for another 45 minutes. Later, the acetone was removed using three methods, namely, hot air oven at $55^{\circ} \mathrm{C}(\mathrm{A})$, magnetic stirrer at $55^{\circ} \mathrm{C}(\mathrm{B})$, and under vacuum at room temperature $(\mathrm{C})$. Then, the epoxy-MWCNTs mixture was mixed with required quantity of hardener by the hand mixing process for $15 \mathrm{~min}$. and poured into the die. Thus, the nanocomposites were prepared once the mold was cured.

2.4. Characterization of Test Samples. The compression test of the sample was carried out as per ASTM D695 [17] using 


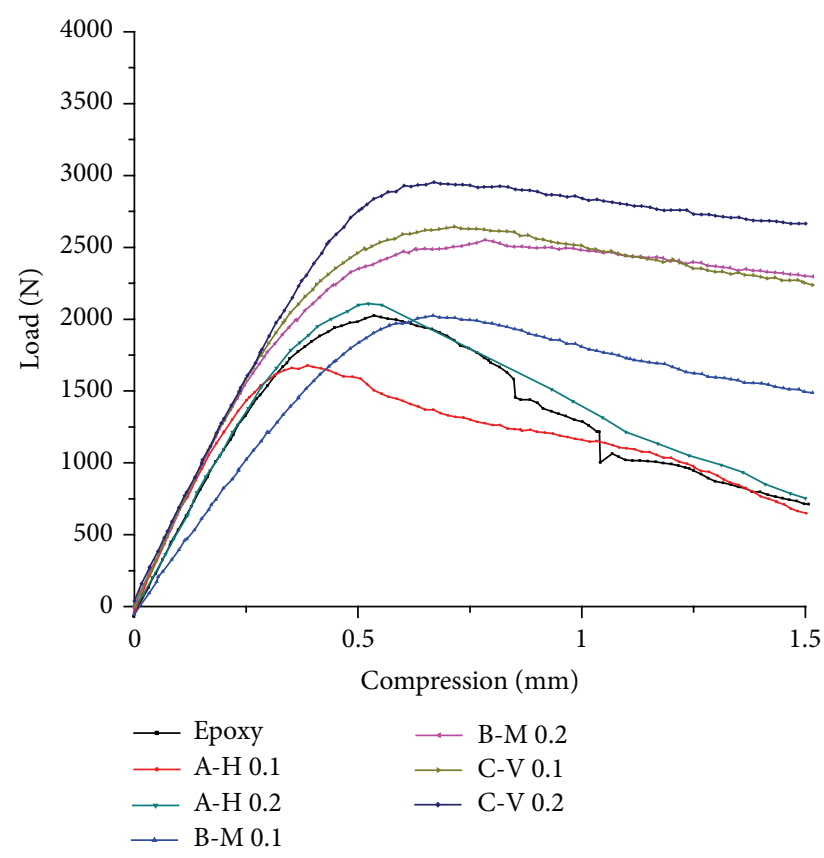

Figure 1: Load versus compression of epoxy nanocomposites.

a servo controlled closed loop Instron 8101. The thermal conductivity of the sample was measured by a KD2 Pro thermal properties analyzer using a dual-probe method. A Netzsch simultaneous thermal analyzer model STA 449 F3 having the DSC resolution of $<1 \mu \mathrm{W}$ and the microbalance resolution of $<1 \mu \mathrm{g}$ was used for calorimetric analysis of the test samples from room temperature to $800^{\circ} \mathrm{C}$ at a heating rate of $10 \mathrm{~K} / \mathrm{min}$. The test samples were prepared from the cured specimens weighing in the range of 5-10 $\mathrm{mg}$. The sample chamber and the furnace chamber were purged with Ar gas before starting the test at 20 and $60 \mathrm{ml} / \mathrm{min}$., respectively. In all cases, three specimens were tested, and the average of the results is reported. The homogeneous dispersion of the MWCNTs in epoxy was also confirmed by the same.

\section{Results and Discussion}

3.1. Mechanical Properties of Epoxy Nanocomposites. The load versus compression plots obtained for test samples processed by different methods are shown in Figure 1. It is observed that the strength of nanocomposites prepared by magnetic stirring and vacuum oven process was increased. However, it was found to be decreased for the sample prepared through hot air oven process compared to that of pure epoxy. It is also observed that the compressive strength of the nanocomposites was found to be increased with concentration of reinforcement irrespective of the processing technique followed.

The compressive strength (CS) and Young's modulus (EM) of test samples against processing conditions are shown in Figures 2 and 3, respectively. It is observed from Figures 2 and 3 that the CS and EM of epoxy were found to be increased with the reinforcement of MWCNTs. However, the CS and $\mathrm{EM}$ of A-H 0.1 were observed to be decreased by 11.4 and

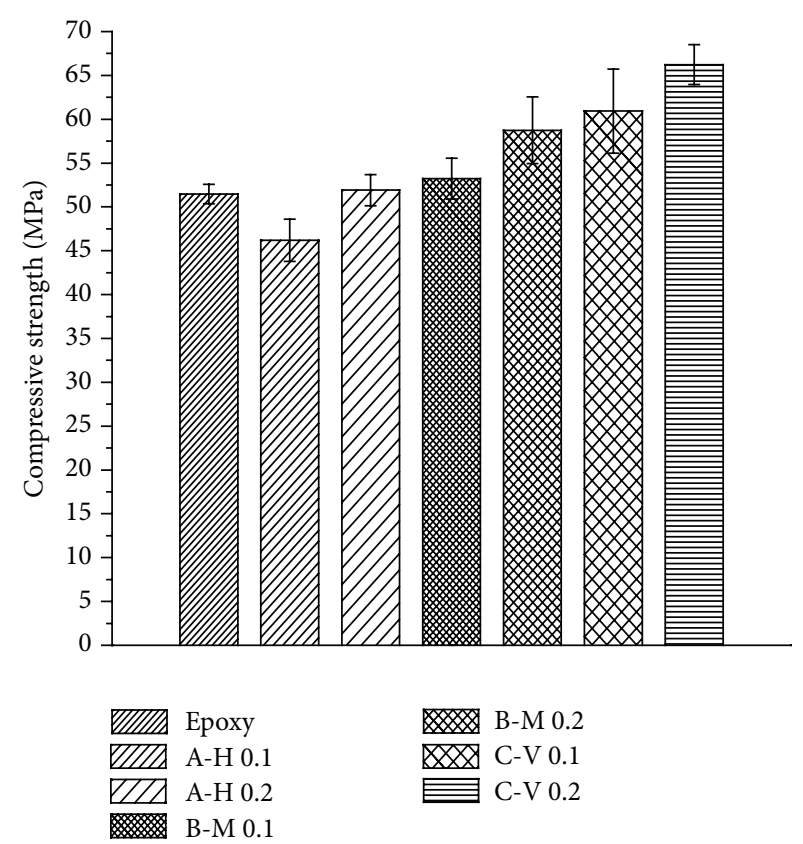

Figure 2: Compressive strength of nanocomposites.

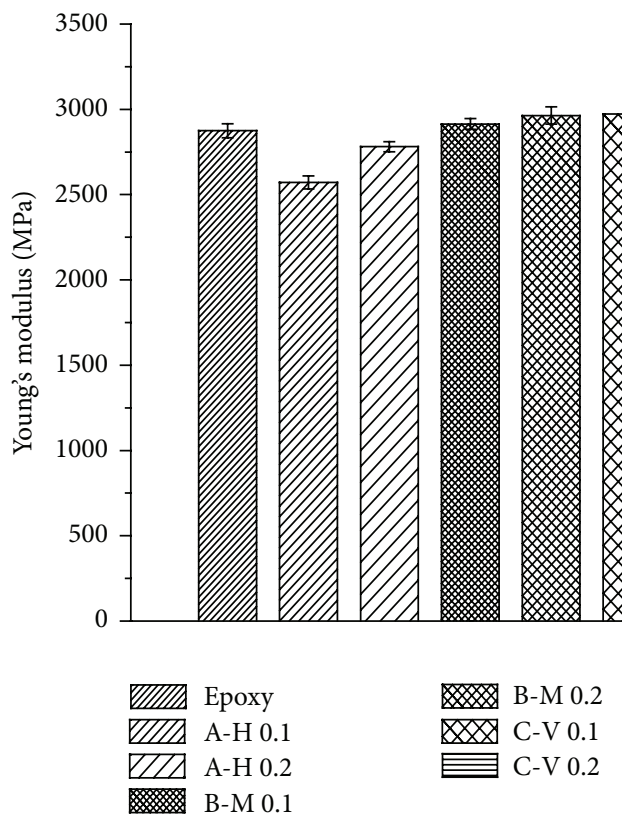

FIGURE 3: Young's modulus of the test samples.

$11.8 \%$, respectively, compared to those of pure epoxy. It is due to the fact that the method of removal of acetone by hot air oven led to destroying the chemical bonding between the MWCNTs and epoxy leading to decreased properties of nanocomposites. However, it was restricted when the MWCNTs' concentration was increased. Amount of heat supplied to evaporate the acetone was not sufficient enough to destroy the chemical bonding between them at $0.2 \mathrm{wt} . \%$ MWCNTs, and, thus, it led to the reduction of CS and EM by only 0.8 and $3.3 \%$, respectively. The sample B-M 


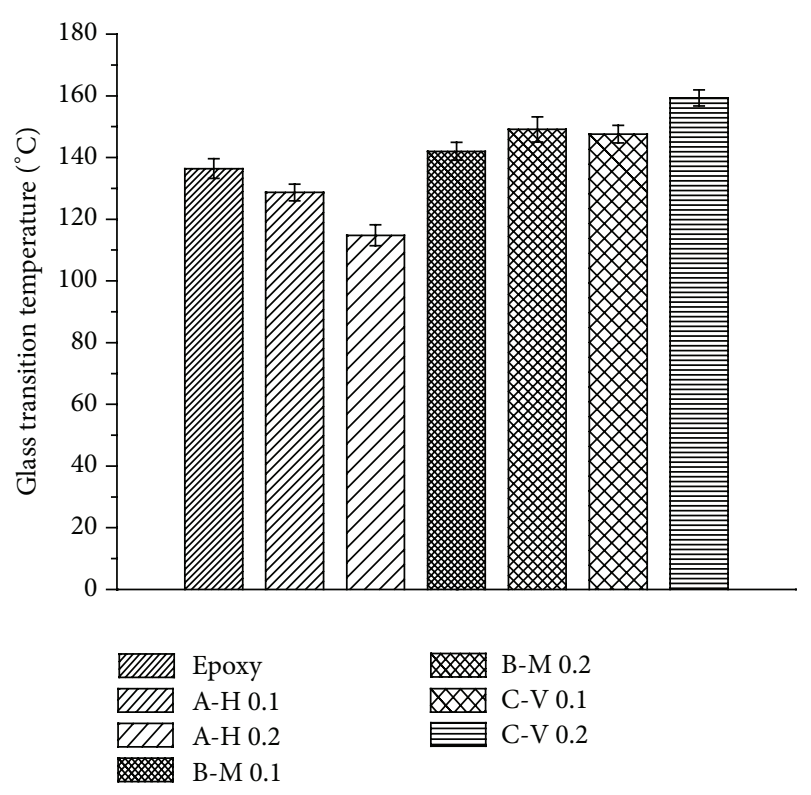

FIGURE 4: Glass transition temperature of the test samples.

0.2 showed the increase of CS and EM of nanocomposites by 14.2 and $3 \%$, respectively, compared to those of pure epoxy resin. The enhancement of mechanical properties is due to the fact that the continuous stirring of the epoxy and MWCNTs in acetone reduced the debonding between them at the evaporation temperature of acetone, and it is expected to ensure the homogeneous dispersion of MWCNTs in epoxy. The sample $\mathrm{C}$ which used the vacuum oven for removing the acetone showed a significant enhancement in the mechanical properties compared to those of the samples A and B. During the vacuum condition at room temperature, the solvent was fully removed compared to the rest of the process. The enhancement of CS and EM of the C-V 0.2 sample was observed to be 39.4 and $10.7 \%$, respectively, compared to pure epoxy. The observed enhancement of EM of epoxy with 0.1 wt.\% MWCNTs was $3.4 \%$, whereas it was reported to be only $2 \%$ for the same reinforcement condition by Starkova et al. [4]. The enhancement of mechanical properties of the nanocomposites was due to homogeneous dispersion of reinforcement and good interaction between the reinforcement and the matrix, which was also confirmed by Gojny and Schulte [18]. A strong physical network between MWCNTs and epoxy was confirmed by the shifting of glass transition temperature $(\mathrm{Tg})$ of the nanocomposites, which is shown in Figure 4, leading to enhanced mechanical properties. The $\mathrm{Tg}$ of $\mathrm{C}-\mathrm{V} 0.2$ was observed to increase by $16.8 \%$ compared to that of pure epoxy confirming the previous observations. Guadagno et al. [19] confirmed the interaction between epoxy and MWCNTs with an increase of Tg and the homogeneous dispersion of the MWCNTs in epoxy. Pillai and Ray [13] confirmed the interaction between epoxy and MWCNTs with the broadening of $\mathrm{Tg}$.

3.2. Thermal Conductivity and Stability of Epoxy Nanocomposites. Figure 5 shows the thermal conductivity of epoxy and its

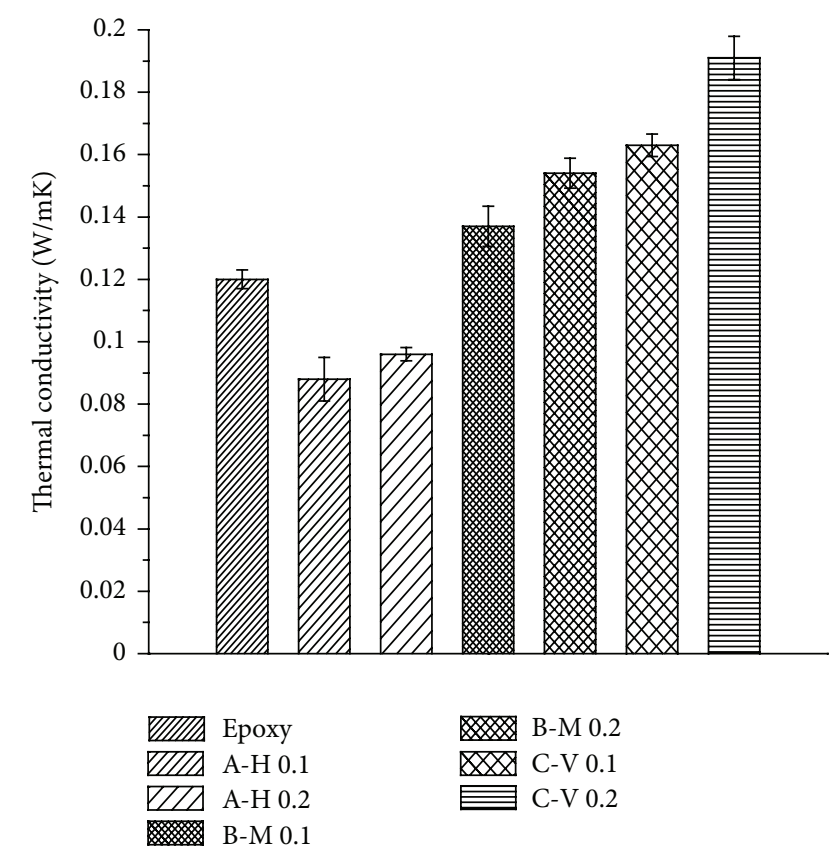

FIGURE 5: Thermal conductivity of nanocomposites.

nanocomposites prepared by different processing conditions, It is observed that the thermal conductivity of the sample A-H 0.1 and A-H 0.2 was found to be $33.4 \%$ and $25 \%$, respectively, lower than that of the pure epoxy, which is due to debonding between the reinforcement and the matrix. It is also observed that the thermal conductivity of samples $\mathrm{B}$ and $\mathrm{C}$ was found to be increased with the reinforcement of MWCNTs. The sample $\mathrm{C}$ showed significant enhancement of thermal conductivity compared to that of samples A and B. The enhancement of thermal conductivity is due to homogeneous dispersion of MWCNTs in epoxy resin and bonding between them. The enhancement of thermal conductivity was found to be $59.2 \%$ for C-V 0.2 compared to pure epoxy. The thermal stability of the sample was measured by the thermogravimetric analysis (TGA), and it is confirmed that the thermal stability of nanocomposites was improved significantly compared to that of pure epoxy, and it is shown in Figure 6. Venkata Ramana et al. [20] also observed the improved thermal stability of epoxy/MWCNTs nanocomposites compared to that of pure epoxy.

\section{Conclusions}

It is concluded that the compressive strength and Young's modulus of nanocomposites were found to be significantly increased for the specimens processed by vacuum drying at room temperature compared to any other method. An improved physical network between MWCNTs and epoxy was confirmed by the enhancement of thermal conductivity and the thermal stability. The Young modulus, compressive strength, and thermal conductivity of the vacuum oven processed sample at $0.2 \mathrm{wt}$.\% of MWCNTs were found to be increased by $10.7,39.4$, and $59.2 \%$, respectively, compared 


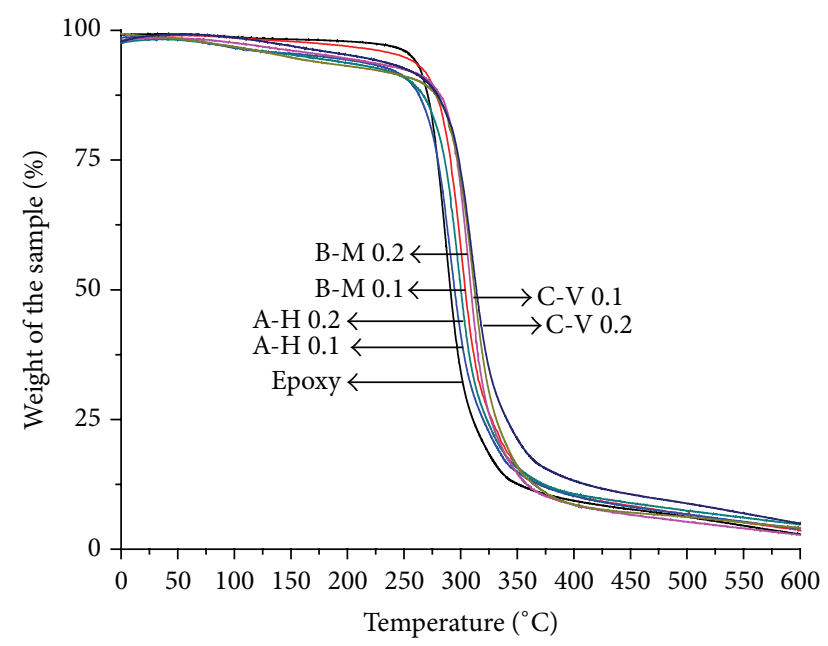

FIgURE 6: Thermal stability of nanocomposites.

to those of pure epoxy. Therefore, a unique method for the preparation of epoxy/MWCNTs nanocomposites is the solvent dispersion technique with vacuum drying process, where maximum enhancement of mechanical and thermal properties, homogeneous, dispersion of reinforcement in the matrix, and good interfacial bonding were obtained.

\section{Abbreviations}

MWCNTs: Multiwalled carbon nanotubes

FTIR: $\quad$ Fourier transform infrared spectroscopy

TGA: Thermogravimetric analysis

DSC: Differential scanning calorimetry

Tg: $\quad$ Glass transition temperature

RT: $\quad$ Room temperature

CS: $\quad$ Compressive strength

EM: $\quad$ Young's modulus.

\section{Acknowledgments}

Technical support given by the staff from the Material Science Lab, Central Instruments Facility, Strength of Materials Lab, and Advanced Manufacturing Lab of IIT Guwahati is highly acknowledged. Funding support given by DBT, India, through Project BT/233/NE/TBP/2011 is also acknowledged.

\section{References}

[1] F. H. Gojny, M. H. G. Wichmann, B. Fiedler, and K. Schulte, "Influence of different carbon nanotubes on the mechanical properties of epoxy matrix composites-a comparative study," Composites Science and Technology, vol. 65, no. 15-16, pp. 23002313, 2005.

[2] M.-F. Yu, B. S. Files, S. Arepalli, and R. S. Ruoff, "Tensile loading of ropes of single wall carbon nanotubes and their mechanical properties," Physical Review Letters, vol. 84, no. 24, pp. 5552$5555,2000$.

[3] M. Kim, Y.-B. Park, O. I. Okoli, and C. Zhang, "Processing, characterization, and modeling of carbon nanotube-reinforced multiscale composites," Composites Science and Technology, vol. 69, no. 3-4, pp. 335-342, 2009.

[4] O. Starkova, S. T. Buschhorn, E. Mannov, K. Schulte, and A. Aniskevich, "Creep and recovery of epoxy/MWCNT nanocomposites," Composites A, vol. 43, pp. 1212-1218, 2012.

[5] H. Mahfuz, S. Zainuddin, M. R. Parker, T. Al-Saadi, V. K. Rangari, and S. Jeelani, "Reinforcement of SC-15 epoxy with CNT/CNF under high magnetic field: an investigation of mechanical and thermal response," Journal of Materials Science, vol. 44, no. 4, pp. 1113-1120, 2009.

[6] L. He and S. C. Tjong, "Carbon nanotube/epoxy resin composite: correlation between state of nanotube dispersion and Zener tunneling parameters," Synthetic Metals, vol. 162, pp. 2277-2281, 2012.

[7] P.-C. Ma, S.-Y. Mo, B.-Z. Tang, and J.-K. Kim, "Dispersion, interfacial interaction and re-agglomeration of functionalized carbon nanotubes in epoxy composites," Carbon, vol. 48, no. 6, pp. 1824-1834, 2010.

[8] N. A. Buang, F. Fadil, Z. A. Majid, and S. Shahir, "Characteristic of mild acid functionalized multiwalled carbon nanotubes towards high dispersion with low structural defects," Digest Journal of Nanomaterials and Biostructures, vol. 7, no. 1, pp. 3339, 2012.

[9] M. Theodore, M. Hosur, J. Thomas, and S. Jeelani, "Influence of functionalization on properties of MWCNT-epoxy nanocomposites," Materials Science and Engineering A, vol. 528, no. 3, pp. 1192-1200, 2011.

[10] N. Yu, Z. H. Zhang, and S. Y. He, "Fracture toughness and fatigue life of MWCNT/epoxy composites," Materials Science and Engineering A, vol. 494, no. 1-2, pp. 380-384, 2008.

[11] M. M. Rahman, S. Zainuddin, M. V. Hosur et al., "Improvements in mechanical and thermo-mechanical properties of eglass/epoxy composites using amino functionalized MWCNTs," Composite Structures, vol. 94, pp. 2397-2406, 2012.

[12] W. Cui, F. Du, J. Zhao et al., "Improving thermal conductivity while retaining high electrical resistivity of epoxy composites by incorporating silica-coated multi-walled carbon nanotubes," Carbon, vol. 49, no. 2, pp. 495-500, 2011.

[13] S. K. Pillai and S. S. Ray, "Epoxy-based carbon nanotubes reinforced composites in advances," in Nanocomposites-Synthesis, Characterization and Industrial Applications, B. Reddy, Ed., pp. 727-792, 2011.

[14] J. G. Park, Q. Cheng, J. Lu et al., "Thermal conductivity of MWCNT/epoxy composites: the effects of length, alignment and functionalization," Carbon, vol. 50, no. 6, pp. 2083-2090, 2012.

[15] K. Esumi, M. Ishigami, A. Nakajima, K. Sawada, and H. Honda, "Chemical treatment of carbon nanotubes," Carbon, vol. 34, no. 2, pp. 279-281, 1996.

[16] M. A. M. Motchelaho, H. Xiong, M. Moyo, L. L. Jewell, and N. J. Coville, "Effect of acid treatment on the surface of multiwalled carbon nanotubes prepared from Fe-Co supported on $\mathrm{CaCO}_{3}$ : correlation with Fischer-Tropsch catalyst activity," Journal of Molecular Catalysis A: Chemical, vol. 335, no. 1-2, pp. 189-198, 2011.

[17] ASTM D 695-10., "Standard Test Method for Compressive Properties of Rigid Plastics," 2010.

[18] F. H. Gojny and K. Schulte, "Functionalisation effect on the thermo-mechanical behaviour of multi-wall carbon nanotube/ epoxy-composites," Composites Science and Technology, vol. 64, no. 15, pp. 2303-2308, 2004. 
[19] L. Guadagno, L. Vertuccio, A. Sorrentino et al., "Mechanical and barrier properties of epoxy resin filled with multi-walled carbon nanotubes," Carbon, vol. 47, no. 10, pp. 2419-2430, 2009.

[20] G. Venkata Ramana, B. Padya, R. Naresh Kumar, K. P. V. Prabhakar, and P. K. Jain, "Mechanical properties of multiwalled carbon nanotubes reinforced polymer nanocomposites," Indian Journal of Engineering and Materials Sciences, vol. 17, no. 5, pp. 331-337, 2010. 

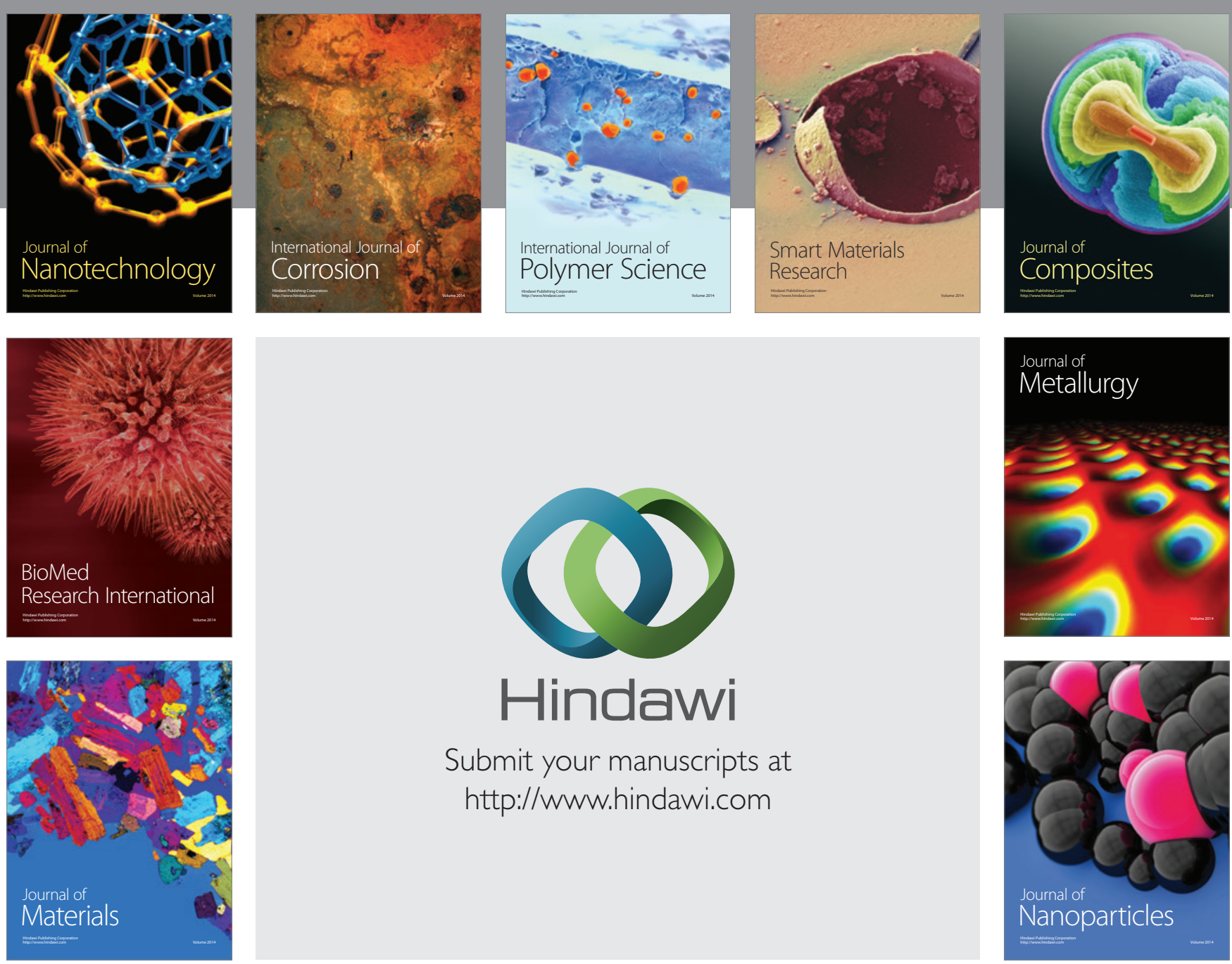

Submit your manuscripts at http://www.hindawi.com
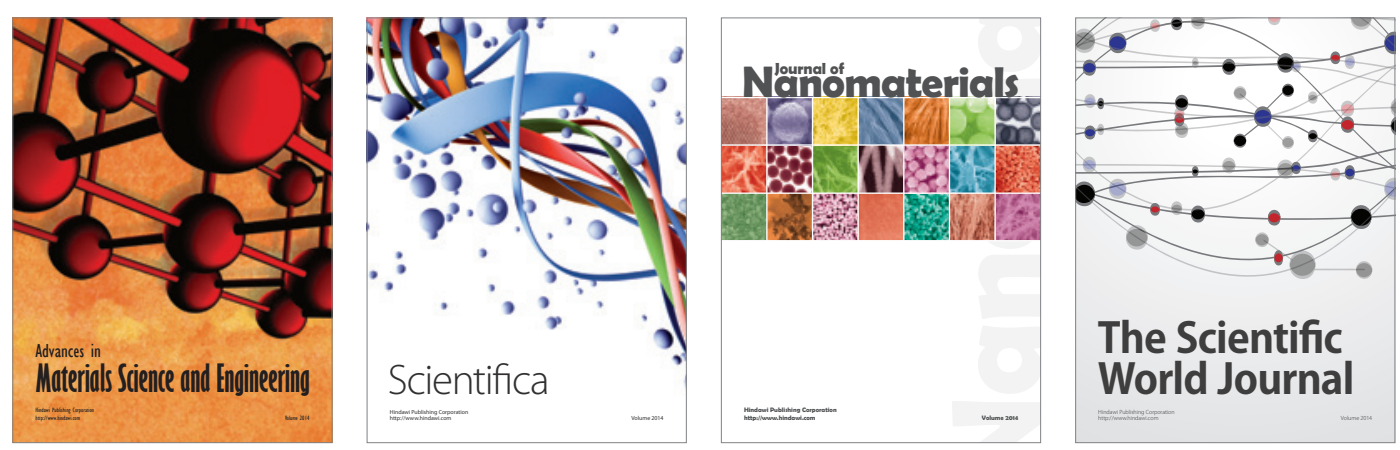

\section{The Scientific World Journal}
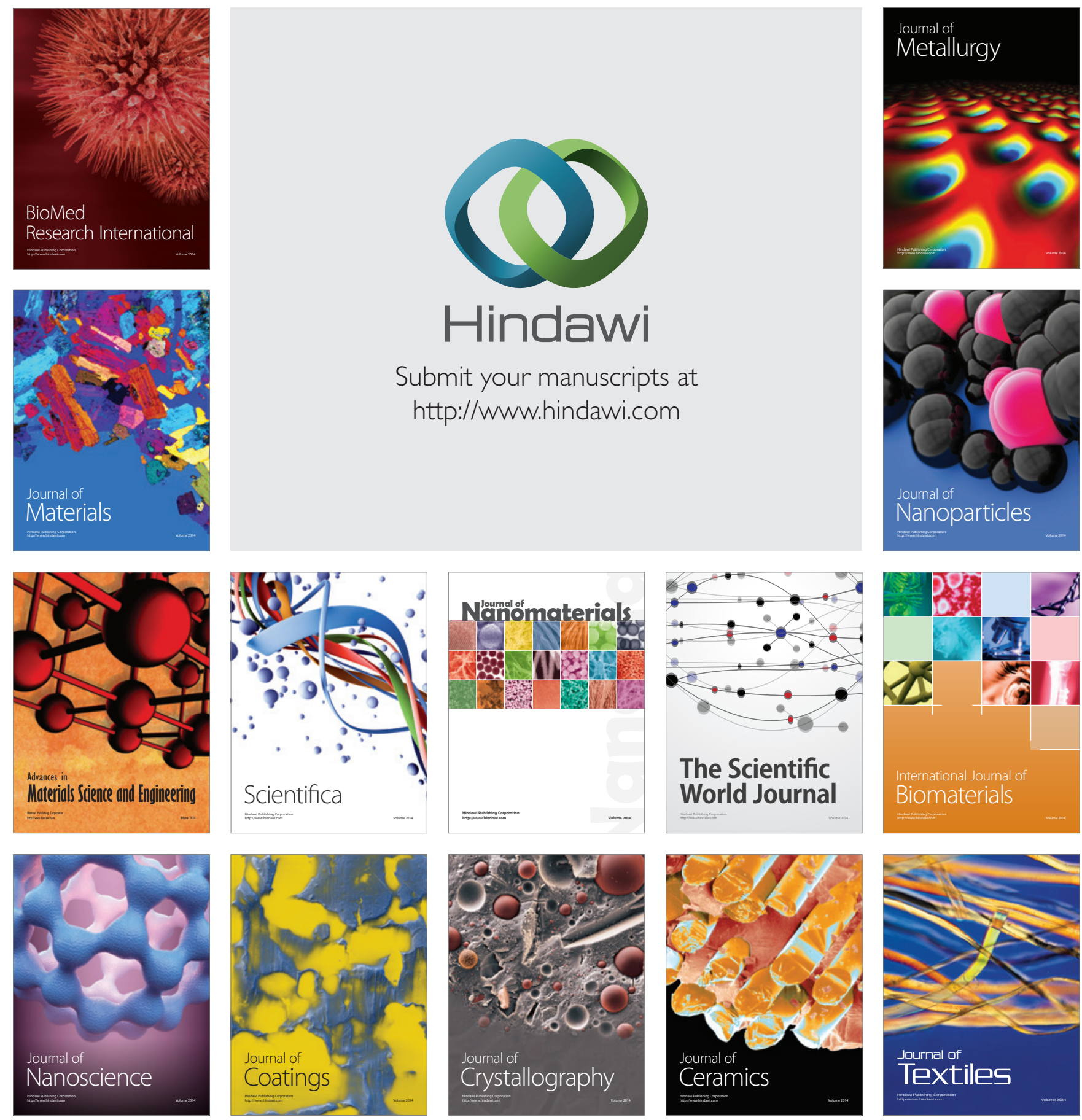